\title{
Efficiency of the centrifugally induced curvature drift instability in AGN winds
}

\author{
Z. Osmanov \\ E. Kharadze Georgian National Astrophysical Observatory, Kazbegi str. 2a, 0106 Tbilisi, Georgia \\ e-mail: z.osmanov@astro-ge.org; osmanov@ph.unito.it \\ Received 4 March 2008 / Accepted 10 June 2008 \\ ABSTRACT

\begin{abstract}
Aims. We investigate the centrifugally driven curvature drift instability to study how field lines twist close to the light cylinder surface of an AGN, through which the free motion of AGN winds can be monitored.

Methods. By studying the dynamics of the relativistic MHD flow close to the light cylinder surface, we derive and solve analytically

Results. Considering the typical values of AGN winds, it is shown that the timescale of the curvature drift instability is far less than plasmas.
\end{abstract} \\ the dispersion relation of the instability by applying a single particle approach based on the centrifugal acceleration. \\ the accretion process timescale, indicating that the present instability is very efficient and might strongly influence processes in AGN
}

Key words. instabilities - magnetohydrodynamics (MHD) - plasmas - galaxies: active - galaxies: nuclei - acceleration of particles

\section{Introduction}

For studying AGN winds the fundamental problem relates to the understanding of a question: how the plasma goes through the Light Cylinder Surface LCS, which is the hypothetical zone where the linear velocity of rotation equals the speed of light. An innermost region of AGNs is characterized by the rotational motion, and it is obvious that this type of motion must affect the plasma dynamics. According to the standard model, the magnetic field due to the frozen-in condition generates plasmas, which consequently flow in the direction of and co-rotate with the field lines. This implies that the plasma particles, which move along quasi-straight magnetic field lines in the nearby area of the LCS, must reach the speed of light. On the other hand, no physical system can maintain such a motion and a certain twisting process of the magnetic field lines must operate on the LCS. In generalizing the work (Machabeli \& Rogava 1994), Rogava et al. (2003) considered curved trajectories and studied the dynamics of a single particle moving along a rotating, curved channel. If the trajectories are given by the Archimedes spiral, then the particles can cross the LCS avoiding the light cylinder problem. An additional step in this investigation is to identify the appropriate mechanism that provides the twisting of the magnetic field lines, giving rise to the shape of the Archimedes spiral, and in turn insures the dynamics is force-free.

In the context of the force-free regime, the light cylinder problem has been studied numerically for pulsars. The investigation developed in Spitkovsky \& Arons (2002) and Spitkovsky (2004) has shown that the plasma can travel through the LCS. This work was based on a current generated by the electric drift (Blandford 2002), which vanished for quasi-neutral plasmas and could not contribute to the dynamics of astrophysical flows, since it had almost equal numbers of positive and negative charges.

Since the innermost region of AGNs rotates, the role of the Centrifugal Force (CF) appears interesting to the study of the relativistic plasma motion. The centrifugally driven outflows have been extensively studied. The study of
Blandford \& Payne (1982) deserves particular attention because these authors discused the possibility that the energy and angular momentum originated in the accretion disk, emphasizing the role of the centrifugal acceleration in this process. Gangadhara \& Lesch (1997) considered the CF in the context of the nonthermal radiation from the spinning AGNs. Generalizing the work of Gangadhara \& Lesch it was shown (Osmanov et al. 2007; Rieger \& Aharonian 2008), that due to the centrifugal acceleration, electrons gain very high energies with Lorentz factors up to $\gamma \sim 10^{8}$. This implies that the energy budget in the AGN winds is very high and if one finds a mechanism for the conversion of at least a small fraction of this energy into a variety of instabilities, there may be interesting consequences for the physics of AGN outflows.

The centrifugal force may drive different types of instabilities. Obviously, the CF that acts on a moving particle changes with time and, in the context of instabilities, plays a role in determining this parameter. Consequently, the corresponding instability is called the parametric instability.

The centrifugally driven parametric instability was first introduced by Machabeli et al. (2005) for the Crab pulsar magnetosphere. We argued that the centrifugal force may cause the separation of charges, leading to the creation of an unstable electrostatic field. Estimating the linear growth rate, it was shown that the instability was extremely efficient. The method developed by Machabeli et al. (2005) was applied to AGN jets (Osmanov 2008) in studying the stability problem of the rotation-induced electrostatic instability and for understanding how efficient is the centrifugal acceleration in this process. Another kind of the instability which might be induced by the CF is the so called Curvature Drift Instability (CDI). Even if the field lines have initially a very small curvature, this curvature might cause a drifting process of plasma, which priduces to the CDI. Osmanov et al. (2008a) considered the two-component relativistic plasma in studying the role of the centrifugal acceleration in the curvature drift instability for pulsar magnetospheres. The investigation demonstrated that the growth rate was higher than pulsar 
spin-down rates by many orders of magnitude, which implied that the CDI was highly efficient. The curvature drift current produces the toroidal component of the magnetic field, which due to the efficient unstable character of the process amplifies rapidly, changing the overall configuration of the magnetic field. This leads to the transformation of field lines into the shape of the Archimedes spiral, when the motion of the particles switches to the so-called force-free regime (Osmanov et al. 2008b) and the plasma goes through the LCS.

To investigate the twisting process of magnetic field lines due to the CDI, we apply the method developed by Osmanov et al. (2008a) and Osmanov et al. (2008b) to AGN winds.

The paper is arranged as follows. In Sect. 2, we introduce the curvature drift waves and derive the dispersion relation. In Sect. 3, the results for typical AGNs are presented and, in Sect. 4, we summarize our results.

\section{Main consideration}

We begin our investigation by considering the two-component plasma consisting of the relativistic electrons with the Lorentz factor $\gamma_{\mathrm{e}} \sim 10^{5-8}$ (Osmanov et al. 2007; Rieger \& Aharonian 2008) and the bulk component (protons) with $\gamma_{\mathrm{b}} \sim 10$. Since we are interested in the twisting process, we suppose that initially the field lines are almost rectilinear to study how this configuration changes in time.

We start by considering the Euler equation governing the dynamics of plasma particles, co-rotating with the straight magnetic-field lines. By applying the method developed by Chedia et al. (1996), owe can show that the Euler equation assume the following form:

$\frac{\partial \boldsymbol{p}_{\alpha}}{\partial t}+\left(\boldsymbol{v}_{\alpha} \nabla\right) p_{\alpha}=-\gamma_{\alpha} \xi \nabla \xi+\frac{q_{\alpha}}{m_{\alpha}}\left(\boldsymbol{E}+\boldsymbol{v}_{\alpha} \times \boldsymbol{B}\right)$,

$\alpha=\{e, b\}$,

where

$\xi \equiv \sqrt{1-\Omega^{2} R^{2} / c^{2}}$,

and $\boldsymbol{p}_{\alpha}$ is the momentum, $\boldsymbol{v}_{\alpha}-$ the velocity, $\gamma_{\alpha}$ is the Lorentz factor of the relativistic particles, and $\boldsymbol{E}$ and $\boldsymbol{B}$ are the electric field and the magnetic induction, respectively. The article charge and the rest mass are denoted by $q_{\alpha}$ and $m_{\alpha}$ respectively. We express the equation of motion in the cylindrical coordinates (see Fig. 1). The first term on the right-hand side of the Euler equation $-\gamma_{\alpha} \xi \nabla \xi$ represents the centrifugal force, which on the light cylinder surface becomes infinity. This means that its overall effect is significant in the nearby zone of the LCS. For describing our physical system, we require the full set of equations, and to close the system, we add to Eq. (1) both, the continuity equation:

$\frac{\partial n_{\alpha}}{\partial t}+\nabla\left(n_{\alpha} \boldsymbol{v}_{\alpha}\right)=0$

and the induction equation:

$\boldsymbol{\nabla} \times B=\frac{1}{c} \frac{\partial \boldsymbol{E}}{\partial t}+\frac{4 \pi}{c} \sum_{\alpha=e, b} \boldsymbol{J}_{\alpha}$,

where $n_{\alpha}$ and $\boldsymbol{J}_{\alpha}$ are the density and the current, respectively.

In the zeroth approximation, the plasma particles are affected only by the centrifugal force. Different species at different positions experience different $\mathrm{CFs}$, which cause the separation of charges, leading to the creation of the additional electromagnetic field considered as the first order term in our equations.

The leading state is characterized by the frozen-in condition, $\boldsymbol{E}+\boldsymbol{v}_{\alpha 0} \times \boldsymbol{B}_{0}=0$, which reduces Eq. (1) into the following form (Machabeli \& Rogava 1994):

$\frac{\mathrm{d} v}{\mathrm{~d} t}=\frac{\Omega^{2} R}{1-\frac{\Omega^{2} R^{2}}{c^{2}}}\left[1-\frac{\Omega^{2} R^{2}}{c^{2}}-\frac{2 v^{2}}{c^{2}}\right]$.

The present equation of motion describing the kinematic behaviour of the single co-rotating particle has the following solution:

$v(t) \equiv v_{\|} \approx c \cos (\Omega t)$

for ultra relativistic cases $(\gamma \gg 1)$ and the following initial conditions: $R(0)=0, v(0) \approx c(v \equiv \mathrm{d} R / \mathrm{d} t)$. Here $v_{\|}$denotes the velocity component along the magnetic field lines.

Since we assume that the magnetic field lines have initially a small curvature, the particles moving radially drift along the $x$ axis (Osmanov et al. 2008a) (see Fig. 1). The afore mentioned drift of charges produces a corresponding current, which inevitably creates the toroidal magnetic field, changing the overall configuration of the field lines. Therefore, the aim of the present work is to study the role of the centrifugally induced curvature drift instability in the twisting process of the magnetic field lines. For this purpose, we linearize the system of equations Eqs. (1)-(3), perturbing all physical quantities around the leading state:

$\Psi \approx \Psi^{0}+\Psi^{1}$,

$\Psi=\{n, \boldsymbol{v}, \boldsymbol{p}, \boldsymbol{E}, \boldsymbol{B}\}$.

We express the perturbation by the following relation:

$\Psi^{1}(t, r) \propto \Psi^{1}(t) \exp [\mathrm{i}(\boldsymbol{k r})]$,

then, from Eqs. (1)-(3) we derive the linearized set of equations governing the CDI:

$\frac{\partial p_{\alpha x}^{1}}{\partial t}-\mathrm{i}\left(k_{x} u_{\alpha}+k_{\phi} v_{\|}\right) p_{\alpha x}^{1}=\frac{q_{\alpha}}{m_{\alpha}} v_{\|} B_{r}^{1}$,

$\frac{\partial n_{\alpha}^{1}}{\partial t}-\mathrm{i}\left(k_{x} u_{\alpha}+k_{\phi} v_{\|}\right) n_{\alpha}^{1}=\mathrm{i} k_{x} n_{\alpha}^{0} v_{\alpha x}^{1}$,

$-\mathrm{i} k_{\phi} c B_{r}^{1}=4 \pi \sum_{\alpha=e, b} q_{\alpha}\left(n_{\alpha}^{0} v_{\alpha x}^{1}+n_{\alpha}^{1} u_{\alpha}\right)$.

By $u_{\alpha}$, we denote the curvature drift velocity along the $x$ axis:

$u_{\alpha}=\frac{\gamma_{\alpha_{0}} v_{\|}^{2}}{\omega_{B} R_{B}}$

where $\omega_{\alpha B}=q_{\alpha} B_{0} / m_{\alpha} c, R_{B}$ is the curvature radius of magnetic field lines, and

$B_{0}=\sqrt{\frac{2 L}{R_{\mathrm{lc}} c^{2}}}$

is the equipartition magnetic induction on the LCS for the leading state, (where $L$ is the luminosity of the AGN and $R_{\mathrm{lc}}=c / \Omega$ is the light cylinder radius). In deriving Eqs. (9)-(11), the wave propagating almost perpendicular to the equatorial plane, was considered and the expression $v_{r}^{1} \approx c E_{x}^{1} / B_{0}$ was taken into account. For simplicity, the set of equations are given in terms of the coordinates of the field line (see Fig. 1). 


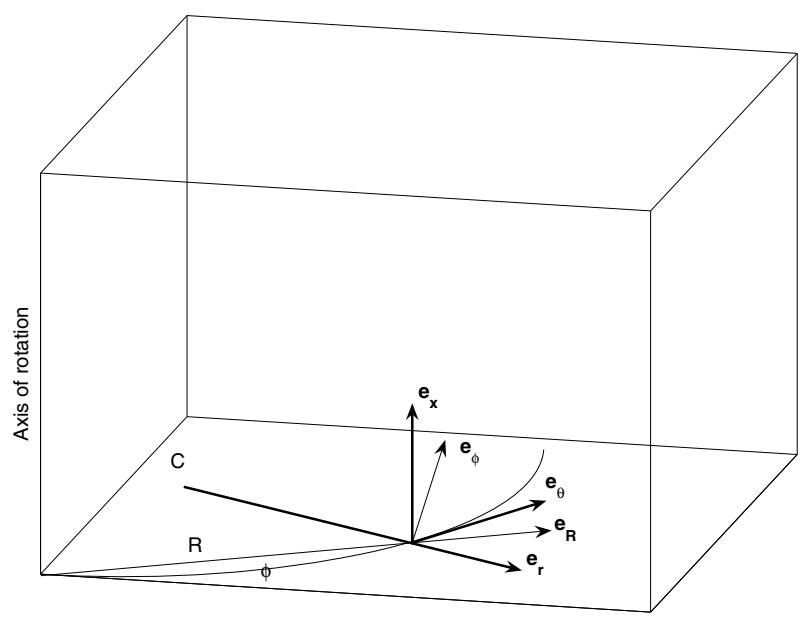

Fig. 1. Two orthonormal bases are considered: i) cylindrical components of unit vectors, $\left(\boldsymbol{e}_{\Phi}, \boldsymbol{e}_{R}, \boldsymbol{e}_{x}\right)$; ii) unit vectors of the system rigidly fixed on each point of the curve, $\left(\boldsymbol{e}_{r}, \boldsymbol{e}_{\theta}, \boldsymbol{e}_{x}\right)$, respectively. $C$ is the center of the curvature. Hereafter, this set of coordinates is referred to as the field line coordinates.

We express $v_{\alpha x}^{1}$ and $n_{\alpha}^{1}$ in the following way:

$v_{\alpha x}^{1} \equiv V_{\alpha x} \mathrm{e}^{\mathrm{i} k \boldsymbol{A}_{\alpha}(t)}$,

$n_{\alpha}^{1} \equiv N_{\alpha} \mathrm{e}^{\mathrm{i} k \boldsymbol{A}_{\alpha}(t)}$,

where

$A_{\alpha x}(t)=\frac{u_{\alpha} t}{2}+\frac{u_{\alpha}}{4 \Omega} \sin (2 \Omega t)$

$A_{\alpha \phi}(t)=\frac{c}{\Omega} \sin (\Omega t)$.

Then, by substituting Eqs. (14) and (15) into Eqs. (9)-(11), it becomes straightforward to solve the system for the toroidal component:

$-\mathrm{i} k_{\phi} c B_{r}^{1}(t)=\sum_{\alpha=e, b} \frac{\omega_{\alpha}^{2}}{\gamma_{\alpha_{0}}} \mathrm{e}^{\mathrm{i} \boldsymbol{k} \boldsymbol{A}_{\alpha}(t)} \int^{t} \mathrm{e}^{-\mathrm{i} \boldsymbol{k} \boldsymbol{A}_{\alpha}\left(t^{\prime}\right)} v_{\|}\left(t^{\prime}\right) B_{r}\left(t^{\prime}\right) \mathrm{d} t^{\prime}$

$+\mathrm{i} \sum_{\alpha=e, b} \frac{\omega_{\alpha}^{2}}{\gamma_{\alpha_{0}}} k_{x} u_{\alpha} \mathrm{e}^{\mathrm{i} \boldsymbol{k} \boldsymbol{A}_{\alpha}(t)} \int^{t} \mathrm{~d} t^{\prime} \int^{t^{\prime \prime}} \mathrm{e}^{-\mathrm{i} \boldsymbol{k} \boldsymbol{A}_{\alpha}\left(t^{\prime \prime}\right)} v_{\|}\left(t^{\prime \prime}\right) B_{r}\left(t^{\prime \prime}\right) \mathrm{d} t^{\prime \prime}$

where $\omega_{\alpha}=e \sqrt{4 \pi n_{\alpha}^{0} / m_{\alpha}}$ is the plasma frequency. After calculating the Fourier transform (see Appendix A), Eq. (18) assumes the form:

$$
\begin{aligned}
& B_{r}(\omega)=-\sum_{\alpha=e, b} \frac{\omega_{\alpha}^{2}}{2 \gamma_{\alpha_{0}} k_{\phi} c} \sum_{\sigma= \pm 1} \sum_{s, n, l, p} \frac{J_{s}\left(g_{\alpha}\right) J_{n}(h) J_{l}\left(g_{\alpha}\right) J_{p}(h)}{\omega+\frac{k_{x} u_{\alpha}}{2}+\Omega(2 s+n)} \\
& \times B_{r}(\omega+\Omega(2[s-l]+n-p+\sigma))\left[1-\frac{k_{x} u_{\alpha}}{\omega+\frac{k_{x} u_{\alpha}}{2}+\Omega(2 s+n)}\right]
\end{aligned}
$$$$
+\sum_{\alpha=e, b} \frac{\omega_{\alpha}^{2} k_{x} u_{\alpha}}{4 \gamma_{\alpha_{0}} k_{\phi} c} \sum_{\sigma, \mu= \pm 1} \sum_{s, n, l, p} \frac{J_{s}\left(g_{\alpha}\right) J_{n}(h) J_{l}\left(g_{\alpha}\right) J_{p}(h)}{\left(\omega+\frac{k_{x} u_{\alpha}}{2}+\Omega(2[s+\mu]+n)\right)^{2}}
$$$$
\times B_{r}(\omega+\Omega(2[s-l+\mu]+n-p+\sigma)),
$$

where

$g_{\alpha}=\frac{k_{x} u_{\alpha}}{4 \Omega}, \quad h=\frac{k_{\phi} c}{\Omega}$.

We note that Eq. (19) is written for $B_{r}(\omega)$, and on the righthand side of the equation, there is the infinite number of components $B_{r}(\omega \pm \Omega), B_{r}(\omega \pm 2 \Omega), \ldots$ etc. This implies that to solve the afore mentioned equation and close the system, we need to add the corresponding expressions for $B_{r}(\omega \pm \Omega), B_{r}(\omega \pm 2 \Omega), \ldots$ etc. However, the system becomes composed of the infinite number of equations, making the task unsolvable. To overcome this problem we have to use a physically reasonable cutoff on theright hand side of the equation (Silin et al. 1970). As is clear from Eq. (19), the considered instability is characterized by the following proper frequency of the curvature drift modes:

$\omega_{0} \approx-\frac{k_{x} u_{\alpha}}{2}$

when the corresponding conditions: $k_{x} u_{\alpha} / 2<0,2 s+n=0$, and $2[s+\mu]+n=0$ are satisfied. As we see, only the resonance terms provide a significant contribution to the result.

We consider the parameters $L \sim 10^{44} \mathrm{erg} / \mathrm{s}, \Omega=3 \times 10^{-5} \mathrm{~s}^{-1}$, $\gamma_{\mathrm{e} 0} \sim 10^{5}, R_{B} \approx R_{\mathrm{lc}} n_{\mathrm{e} 0} \sim 0.001 \mathrm{~cm}^{-3}$ typical for AGN winds. Then, examining the curvature drift waves with $\lambda \sim R_{\mathrm{lc}}$ ( $\lambda=2 \pi / k$ is the wave length), we can show that $\left|k_{x} u_{\mathrm{e}} / 2\right| \sim$ $10^{-12} \mathrm{~s}^{-1} \ll \Omega$, where, it is assumed that $k_{x}<0$ and $u_{\mathrm{e} 0}>0$, otherwise the frequency becomes negative. Therefore, all terms with non-zero values of $\Omega(2 s+n)$ and $\Omega(2[s+\mu]+n)$ oscillate rapidly and do not contribute to the final result. Consequently, the only terms that influence the solution of Eq. (19) are the leading terms, the contribution of which simplifies the equation (Osmanov et al. 2008a) (see Appendix B):

$\left(\omega+\frac{k_{x} u_{\mathrm{e}}}{2}\right)^{2} \approx \sum_{\sigma, \mu= \pm 1} \sum_{s, l} \Xi_{\mu} J_{s}(g) J_{n^{\prime}(s, \mu)}(h) J_{l}(g) J_{p^{\prime}(l, \sigma)}(h)$

where

$\Xi_{0}=2 \Xi_{ \pm 1}=\frac{\omega_{\mathrm{e}}^{2} k_{x} u_{\mathrm{e}}}{2 \gamma_{\mathrm{ep}_{0}} k_{\theta} c}$,

$n^{\prime}=-2(s+\mu), \quad p^{\prime}=-2 l+\sigma, \quad g \equiv g_{\mathrm{e}}$.

We note, that Eq. (21) does not contain the bulk components because, as a direct calculation shows, their contribution is negligible with respect to the terms corresponding to the relativistic electrons. Expressing the frequency in terms of its real and imaginary parts, $\omega \equiv \omega_{0}+\mathrm{i} \Gamma$, it is straightforward to show, from Eq. (21), that the increment of the CDI is given by:

$\Gamma \approx\left[\sum_{\sigma, \mu= \pm 1} \sum_{s, l} \Xi_{\mu} J_{s}(g) J_{-2(s+\mu)}(h) J_{l}(g) J_{-2 l+\sigma}(h)\right]^{\frac{1}{2}}$.

\section{Results}

We investigate the efficiency of the CDI in AGN winds. The behaviour of the growth rate is considered in terms of the wavelength, the density of relativistic electrons, their Lorentz factors and the AGN bolometric luminosity.

In studying the behaviour of the instability as a function of the wavelength, we examine the typical AGN parameters: $M_{\mathrm{BH}}=10^{8} \times M_{\odot}, \Omega=5 \times 10^{-5} \mathrm{~s}^{-1}$ and $L=10^{44} \mathrm{erg} / \mathrm{s}$, where 


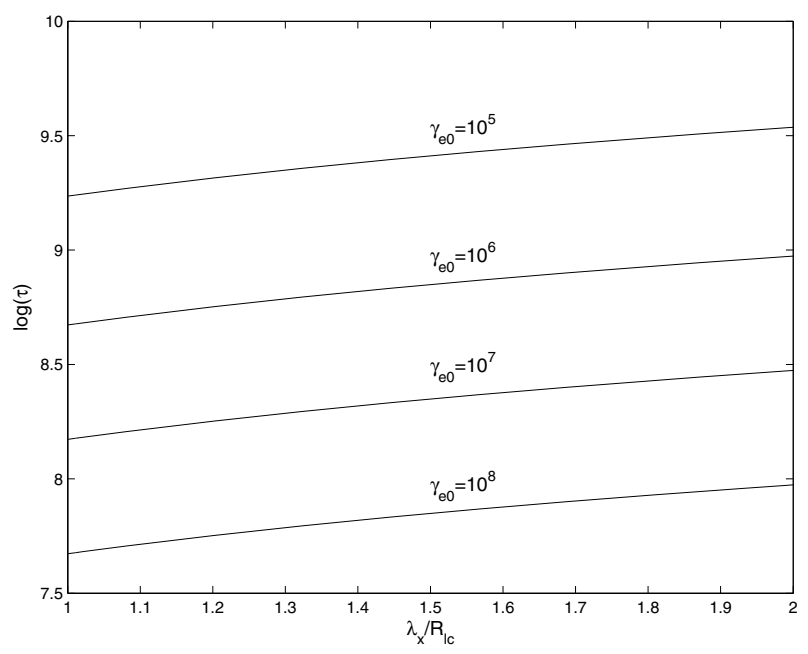

Fig. 2. The dependence of logarithm of the instability timescale on the normalized wave length. The set of parameters is $\gamma_{\mathrm{e} 0}=$ $\left\{10^{5} ; 10^{6} ; 10^{7} ; 10^{8}\right\}, R_{B} \approx R_{\mathrm{lc}}, n_{\mathrm{e} 0}=0.001 \mathrm{~cm}^{-3}, \lambda_{\phi}=100 R_{\mathrm{lc}}$ and $L / L_{\mathrm{E}}=0.01$.

$M_{\mathrm{BH}}$ is the AGN mass, $M_{\odot}$ is the solar mass and $L$ is the bolometric luminosity of the AGN.

We consider Eq. (23) and plot the logarithm of the instability timescale

$\tau \equiv \frac{1}{\Gamma}$

versus the wavelength normalized by the light cylinder radius. The present consideration is based on the centrifugal acceleration. As shown by Osmanov et al. (2007), due to the CF, the relativistic particles can reach very high Lorentz factors. For this purpose, it is reasonable to investigate the efficiency of the instability in terms of the wavelength but for different values of Lorentz factors. Figure 2 shows the mentioned behaviour for the following parameters $\gamma_{\mathrm{e} 0}=\left\{10^{5} ; 10^{6} ; 10^{7} ; 10^{8}\right\}, R_{B} \approx R_{\mathrm{lc}}$, $n_{\mathrm{e} 0}=0.001 \mathrm{~cm}^{-3}, \lambda_{\phi} \equiv 2 \pi / k_{\phi}=100 R_{\mathrm{lc}}$, and $L / L_{\mathrm{E}}=0.01$, where $L_{\mathrm{E}}=10^{46} \mathrm{erg} / \mathrm{s}$ is the Eddington luminosity for the given AGN mass. Different curves correspond to different values of Lorentz factors. As it is clear from the plots, the timescale is a continuously increasing function of $\lambda_{x}\left(\equiv 2 \pi / k_{x}\right)$, which is a direct consequence of Eqs. (22) and (23). As we see from Eq. (22), $\Xi_{0, \pm 1} \sim 1 / \lambda_{x}$, combining with Eq. (23) provides $\Gamma \sim 1 / \sqrt{\lambda_{x}}$. Therefore, the larger the initial perturbation wave length, the lower the instability timescale and consequently the less the CDI efficiency. For the given range of $\lambda_{x}$ and different values of $\gamma_{\mathrm{e} 0}$, the CDI time scale varies from $\sim 10^{7} \mathrm{~s}\left(\lambda_{x} / R_{\mathrm{lc}}=1, \gamma_{\mathrm{e} 0}=10^{8}\right)$ to $\sim 10^{9}\left(\lambda_{x} / R_{\mathrm{lc}}=2, \gamma_{\mathrm{e} 0}=10^{5}\right)$.

Since the CDI growth rate depends on the plasma frequency (see Eqs. (23), (22)), which is in turn a function of the density, it is obvious that the instability timescale must be influenced by the density of relativistic electrons in the AGN winds. In Fig. 3 the plots of $\log (\tau)$ versus the AGN wind density illustrate that the timescale is a continuously decreasing function of $n_{\mathrm{e} 0} / n_{\mathrm{m}}$. The set of parameters is the same as in Fig. 2, apart from $\lambda_{x}=R_{\mathrm{lc}}$ and $n_{\mathrm{e} 0} / n_{\mathrm{m}} \in\{0.001,1\}$. Here, $n_{\mathrm{e} 0}$ is normalized by the intergalactic medium density, $n_{\mathrm{m}} \approx 1 \mathrm{~cm}^{-3}$. As we see from the figure, $\tau$ varies from $\sim 10^{9} \mathrm{~s}\left(n_{\mathrm{e} 0} / n_{\mathrm{m}}=0.001, \gamma_{\mathrm{e} 0}=10^{5}\right)$ to $\sim 10^{6} \mathrm{~s}$ $\left(n_{\mathrm{e} 0} / n_{\mathrm{m}}=1, \gamma_{\mathrm{e} 0}=10^{8}\right)$.

In Fig. 4, the plot of $\log (\tau)$ versus $\log \left(\gamma_{\mathrm{e} 0}\right)$ is shown for different luminosities. The set of parameters is the same as in Fig. 2 apart from the continuous range of $\gamma_{\mathrm{e} 0} \in\left\{10^{5} ; 10^{8}\right\}$, some values of the luminosity $L / L_{\mathrm{E}}=\{0.01 ; 0.05 ; 0.3 ; 1\}$, and $\lambda_{x}=R_{\mathrm{lc}}$.

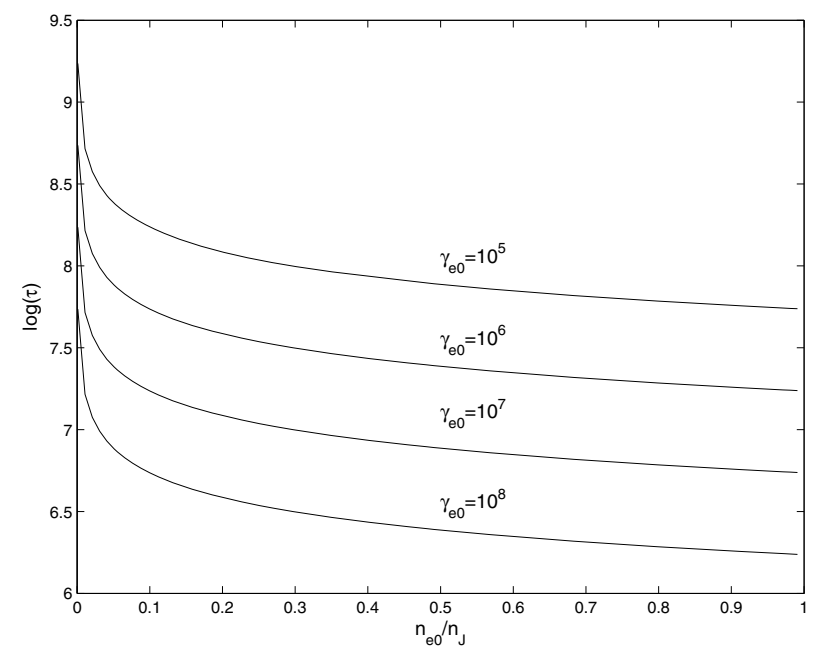

Fig. 3. The dependence of logarithm of the instability timescale on the density normalized by the medium density. The set of parameters is $\gamma_{\mathrm{e} 0}=\left\{10^{5} ; 10^{6} ; 10^{7} ; 10^{8}\right\}, R_{B} \approx R_{\mathrm{lc}}, \lambda_{\mathrm{e} 0}=R_{\mathrm{lc}}, \lambda_{\phi}=100 R_{\mathrm{lc}}$ and $L / L_{\mathrm{E}}=$ 0.01 .

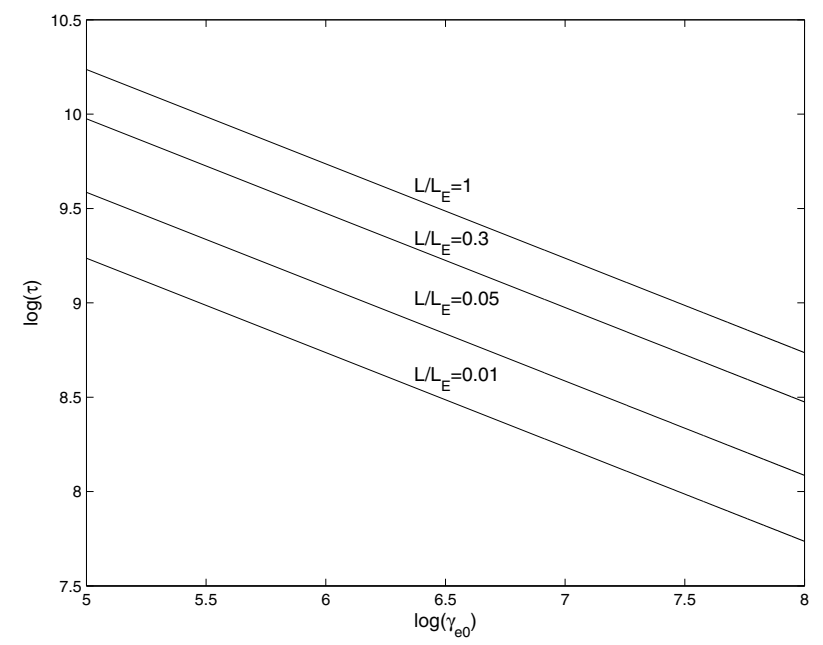

Fig. 4. The dependence of logarithm of the instability time scale on $\log \left(\gamma_{\mathrm{e} 0}\right)$. The set of parameters is $R_{B} \approx R_{\mathrm{lc}}, n_{\mathrm{e} 0} / n_{\mathrm{m}}=0.001, \lambda_{\mathrm{e} 0}=R_{\mathrm{lc}}$, $\lambda_{\phi}=100 R_{\mathrm{lc}}$ and $L / L_{\mathrm{E}}=\{0.01 ; 0.05 ; 0.3 ; 1\}$.

The figure shows the continuously decreasing behaviour of the instability timescale, which is a natural consequence of the fact that the more energetic electrons induce the curvature drift instability more efficiently. From Eq. (12) it is clear that the drift velocity is proportional to the Lorentz factor of the particle, and hence the corresponding instability is more efficient, producing the decrease in $\log (\tau)$. As we see, the instability timescale varies from $\sim 10^{10} \mathrm{~s}\left(\gamma_{\mathrm{e} 0}=10^{5}, L / L_{\mathrm{E}}=1\right)$ to $\sim 10^{8} \mathrm{~s}\left(\gamma_{\mathrm{e} 0}=10^{7}\right.$, $\left.L / L_{\mathrm{E}}=0.01\right)$. On the other hand, the plots for different luminosities illustrates another property of $\tau$ : by increasing the luminosity of the AGN, the corresponding instability becomes less efficient.

To observe this particular feature more clearly, we consider how in Fig. 5 the dependence of $\log (\tau)$ on $L / L_{\mathrm{E}}$ is clearly evident for different values of densities. From the plots, it is seen that by increasing the luminosity, the timescale continuously increases. This behaviour follows from the fact that the bigger the luminosity, the larger the magnetic field (see Eq. (13)) and hence the lower the drift velocity, producing the less efficient CDI shown in the figure. By considering larger values of densities, 


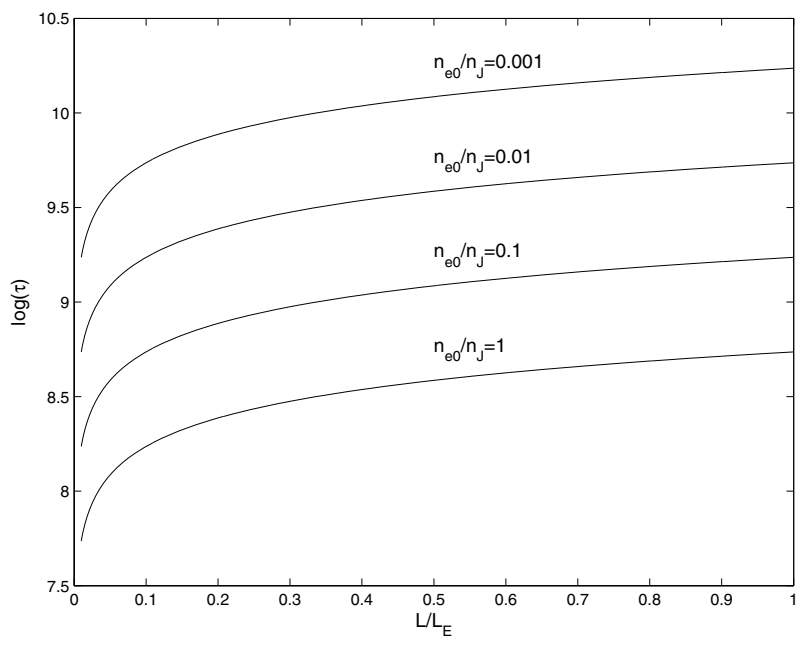

Fig. 5. The dependence of logarithm of the instability time scale on $\log \left(\gamma_{\mathrm{e} 0}\right)$. The set of parameters is $R_{B} \approx R_{\mathrm{lc}}, n_{\mathrm{e} 0} / n_{\mathrm{m}}=$ $\{0.001 ; 0.01 ; 0.1 ; 1\}, \lambda_{\mathrm{e} 0}=R_{\mathrm{lc}}, \lambda_{\phi}=100 R_{\mathrm{lc}}$ and $L / L_{\mathrm{E}}=0.01$.

the CDI becomes more efficient, which we explained already while considering Fig. 3. For the afore mentioned area of quantities (see Fig. 5), the timescale varies from $\sim 10^{7} \mathrm{~s}\left(L / L_{\mathrm{E}}=0.01\right.$, $\left.n_{\mathrm{e} 0} / n_{J}=1\right)$ to $\sim 10^{10} \mathrm{~s}\left(L / L_{\mathrm{E}}=1, n_{\mathrm{e} 0} / n_{J}=0.001\right)$.

We observe from the present investigation that the instability timescale varies in the following range: $\tau \in\left\{10^{6} ; 10^{10}\right\} \mathrm{s}$. To specify how efficient the CDI is, it is pertinent to examine an accretion process, estimate its corresponding evolution timescale, and compare this value with that of the CDI.

Considering the problem of fuelling AGNs, King \& Pringle (2007) showed that the self-gravitating mass in accretion flows can be estimated by the following expression:

$$
\begin{aligned}
M_{\mathrm{sg}}= & 2.76 \times 10^{5}\left(\frac{\eta}{0.03}\right)^{-2 / 27}\left(\frac{\epsilon}{0.1}\right)^{-5 / 27}\left(\frac{L}{0.1 L_{\mathrm{E}}}\right)^{5 / 27} \\
& \times\left(\frac{M_{\mathrm{BH}}}{10^{8} M_{\odot}}\right)^{23 / 27} M_{\odot},
\end{aligned}
$$

where $\eta$ and $\epsilon$ are Shakura-Sunyaev viscosity parameter (Shakura \& Sunyaev 1973) and the accretion parameter, respectively. The latter can be defined by means of the accretion mass rate $\dot{M}$ and the luminosity:

$\epsilon \equiv \frac{L}{\dot{M} c^{2}}$

Then, defining the accretion timescale to be $t_{\mathrm{evol}} \equiv M_{\mathrm{sg}} / \dot{M}$ we can reduce this to (King \& Pringle 2007):

$$
\begin{aligned}
t_{\mathrm{evol}}= & 3.53 \times 10^{13}\left(\frac{\eta}{0.03}\right)^{-2 / 27}\left(\frac{\epsilon}{0.1}\right)^{22 / 27}\left(\frac{L}{0.1 L_{\mathrm{E}}}\right)^{-22 / 27} \\
& \times\left(\frac{M_{\mathrm{BH}}}{10^{8} M_{\odot}}\right)^{-4 / 27} \mathrm{s.}
\end{aligned}
$$

As is clear from Eq. (27), the accretion evolution timescale depends on two major AGN parameters, the luminosity $(L)$ and the AGN mass $\left(M_{\mathrm{BH}}\right)$. Considering Eq. (27), it is reasonable to investigate $t_{\mathrm{evol}}$ versus $L$ and $M_{\mathrm{BH}}$. For this purpose, we consider the possible maximum area of the parametric space, $L-M_{\mathrm{BH}}$ studying the behaviour of $t_{\mathrm{evol}}$ for the typical accretion disk parameters $\eta=0.03$ and $\epsilon=0.1$.

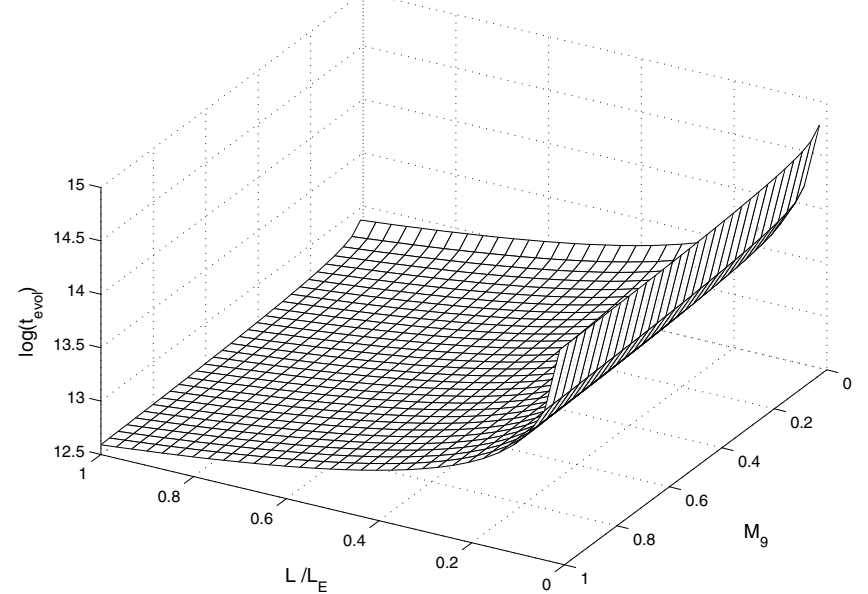

Fig. 6. The behaviour of logarithm of the evolution timescale versus $M_{9}$ and $L / L_{\mathrm{E}}$. The set of parameters is $\eta=0.03, \epsilon=0.1$.

In Fig. 6, we show the two-dimensional surface of logarithm of the evolution timescale. The variables are in the following range: $M_{9} \equiv M_{\mathrm{BH}} /\left(10^{9} \times M_{\odot}\right) \in\{0.01 ; 1\}$ and $L / L_{\mathrm{E}} \in$ $\{0.01 ; 1\}$. For plotting the figure, we took into account that, according to the observations, AGN masses vary in the following range: $M_{\mathrm{BH}} \in\left\{10^{6} ; 10^{9}\right\} \times M_{\odot}$ (Nelson 2000). As is clear from Fig. $6, t_{\mathrm{evol}}$ is a continuously decreasing function of $M_{9}$ and $L / L_{\mathrm{E}}$. The minimum value of the evolution timescale $\left(\sim 10^{12} \mathrm{~s}\right)$, when the accretion process is extremely efficient corresponds to $M_{9}=1$ and $L / L_{\mathrm{E}}=1$, whereas the maximum value, approximately $\sim 10^{15} \mathrm{~s}$, corresponds to the following pair of variables $M_{9}=0.001$ and $L / L_{\mathrm{E}}=0.01$.

To understand how efficient the curvature drift instability is, we have to compare the corresponding timescale with the evolution timescale. As has been found, depending on physically reasonable parameters, $\tau$ varies in the range $\sim\left(10^{6}-10^{10}\right) \mathrm{s}$, whereas the sensible area of $t_{\mathrm{evol}}$ is $\sim\left(10^{12}-10^{15}\right) \mathrm{s}$. Therefore, the instability timescale is less than the evolution timescale of the accretion by many orders of magnitude, which implies that the linear stage of the CDI is extremely efficient.

The twisting process of magnetic field lines requires a certain amount of energy and it is natural to study the energy budget of this process. For this reason we have to introduce the maximum of the possible luminosity $L_{\max }=\dot{M} c^{2}$ and compare this with the "luminosity" corresponding to the reconstruction of the magnetic field configuration $L_{\mathrm{m}} \equiv \Delta E_{\mathrm{m}} / \Delta t \approx \Delta E_{\mathrm{m}} / \tau$, where $\Delta E_{\mathrm{m}}$ is the variation in the magnetic energy due to the curvature drift instability.

We consider a AGN of the luminosity, $L=10^{45} \mathrm{erg} / \mathrm{s}$, then, by applying Eq. (26) and taking into account that $\epsilon=0.1$, we show that the accretion provides the following maximum value:

$L_{\max }=10^{46} \mathrm{erg} / \mathrm{s}$.

On the other hand, if the process of sweepback is realistic, the magnetic "luminosity" cannot exceed $L_{\max }$. Since this process occurs in the nearby zone of the LCS, the effective spatial volume can be estimated by the following expression:

$\Delta V \approx R_{\mathrm{lc}}^{2} \Delta R=R_{\mathrm{lc}}^{3} \kappa$,

where $\kappa \equiv \Delta R / R_{\mathrm{lc}} \ll 1$ represents the non-dimensional thickness of the considered spatial zone. Taking Eq. (29) into account the expression of the magnetic "luminosity" reduces to:

$L_{\mathrm{m}}=\frac{B_{r}^{2}}{4 \pi \tau} R_{\mathrm{lc}}^{3} \kappa$ 


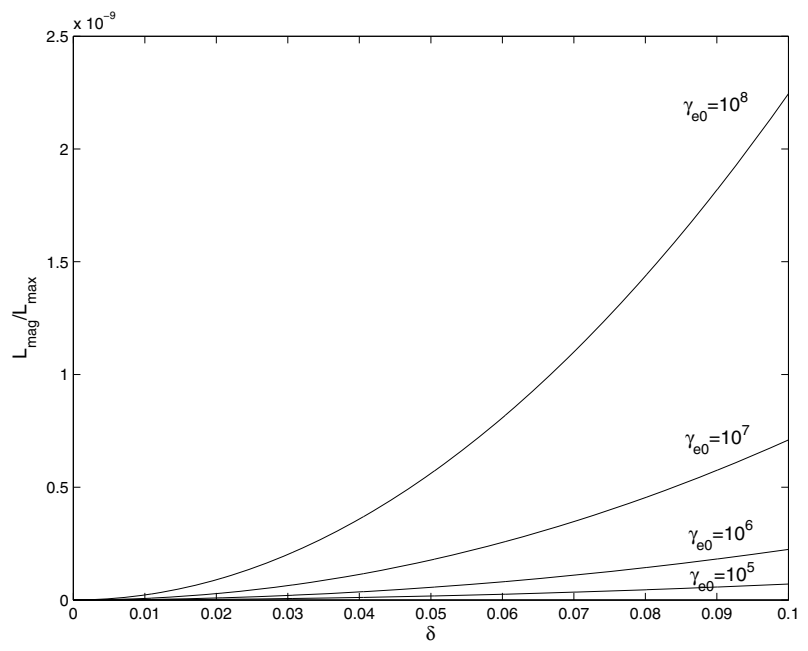

Fig. 7. The dependence of $L_{\mathrm{m}} / L_{\max }$ versus $\delta$. The set of parameters is $\gamma_{\mathrm{e} 0}=\left\{10^{5} ; 10^{6} ; 10^{7} ; 10^{8}\right\}, R_{B} \approx R_{\mathrm{lc}}, n_{\mathrm{e} 0}=0.001 \mathrm{~cm}^{-3}, \lambda_{\phi}=100 R_{\mathrm{lc}}$, $\lambda_{x}=R_{\mathrm{lc}}$ and $L=10^{45} \mathrm{erg} / \mathrm{s}$.

where $B_{r}$, due to the CDI, varies with time as:

$B_{r}=B_{r}^{0} \mathrm{e}^{t / \tau}$

where $B_{r}^{0}$ is the initial perturbation of the toroidal component of magnetic field.

We introduce the initial non-dimensional perturbation, $\delta$, defined to be $\delta \equiv B_{r}^{0} / B_{0}$, where by $B_{0}$ we denote the induction of the magnetic field in the leading state (see Eq. (31)). By considering the following set of parameters $\gamma_{\mathrm{e} 0}=\left\{10^{5} ; 10^{6} ; 10^{7} ; 10^{8}\right\}$, $R_{B} \approx R_{\mathrm{lc}}, n_{\mathrm{e} 0}=0.001 \mathrm{~cm}^{-3}, \lambda_{\phi}=100 R_{\mathrm{lc}}, \lambda_{x}=R_{\mathrm{lc}}$ and $L=10^{45} \mathrm{erg} / \mathrm{s}$, we plot the behaviour of $L_{\mathrm{m}} / L_{\max }$ versus the initial perturbation for the characteristic timescale $(t \approx \tau)$. As we can see from Fig. $7, L_{\mathrm{m}} / L_{\max }$ varies from $\sim 0(\delta=0)$ to $\sim 2.3 \times 10^{-9}\left(\delta=0.1, \gamma_{\mathrm{e} 0}=10^{8}\right)$. Therefore, the maximum luminosity, and therefore the total luminosity budget, exceeds, by many orders of magnitude, the magnetic luminosity required for the twisting of the field lines. This means that only a tiny fraction of the total energy goes to the sweepback, making this process feasible.

\section{Summary}

We summarize the principal steps and conclusions of our study to be:

1. Considering the relativistic two-component plasma for AGN winds, the centrifugally driven curvature drift instability has been studied.

2. Taking into account a quasi single approach for the particle dynamics, we linearized the Euler continuity and induction equations. The dispersion relation characterizing the parametric instability of the toroidal component of the magnetic field has been derived.

3. By considering the proper frequency of the curvature drift modes, the corresponding expression of the instability increment has been obtained for the light cylinder region.
4. The efficiency of the CDI has been investigated by adopting four physical parameters, namely: the wavelength, flow density and Lorentz factors of electrons, and the luminosity of AGNs.

5. By considering the evolution process of accretion, the corresponding timescale has been estimated for a physically reasonable area in the parametric space $L-M_{\mathrm{BH}}$. It was shown that the instability timescale was lower by many orders of magnitude than the evolution timescale, indicating extremely high efficiency of the CDI.

6. Examining the instability from the point of view of the energy budget, we have seen that the sweepback of the magnetic field lines requires only a small fraction of the total energy, which means that the CDI is a realistic process.

An important restriction of the present work is that to describe the plasma kinematics a single particle approach has been applied. On the other hand, it is natural to suppose that the collective phenomena must strongly influence the overall kinematic picture of the plasma motion. For understanding how the collective phenomena changes the instability, we have to generalize the present model to a more realistic astrophysical scenario.

The next limitation is related to the fact that the magnetic field lines were supposed to be quasi rectilinear, whereas in real astrophysical situations the field lines might be initially curved. This particular case also needs to be studied by generalizing the present model.

In this paper, the field lines located in the equatorial plane have been considered, although in realistic situations, the magnetic field lines also might be inclined with respect to the equatorial plane. Therefore, it is essential to examine this particular case as well and see how the efficiency of the CDI changes, when the field line's inclination with respect to the equatorial plane is taken into account.

Acknowledgements. I thank professor G. Machabeli for valuable discussions. The research was supported by the Georgian National Science Foundation grant GNSF/ST06/4-096.

\section{References}

Blandford, R. D. 2002, Luml. Conf., 381B

Blandford, R. D., \& Payne, D. G. 1982, MNRAS, 199, 883

Chedia, O. V., Kahniashvili, T. A., Machabeli, G. Z., \& Nanobashvili, I. S. 1996, Astrophys. Space Sci., 239, 57

Gangadhara, R. T., \& Lesch, H. 1997, A\&A, 323, L45

King, A. R., \& Pringle, J. E. 2007, MNRAS, 377, 25

Machabeli, G., \& Rogava, A. D. 1994, Phys. Rev. A, 50, 98

Machabeli, G., Osmanov, Z., \& Mahajan, S. 2005, Phys. Plasmas, 12, 062901

Nelson, C. H. 2000, ApJ, 544, L91

Osmanov, Z. 2008, Phys. Plasmas, 15, 032901

Osmanov, Z., Dalakishvili, Z., \& Machabeli, Z. 2008a, MNRAS, 383, 1007

Osmanov, Z., Shapakdze, D., \& Machabeli, G. 2008b, ApJ, submitted

Osmanov, Z., Rogava, A. S., \& Bodo, G. 2007, A\&A, 470, 395

Rieger, F. M., \& Aharonian, F. A. 2008, A\&A, 479, 5

Rogava, A. D., Dalakishvili, G., \& Osmanov, Z. 2003, Gen. Rel. Grav., 35, 1133

Shakura, N. I., \& Sunyaev, R. A. 1973, A\&A, 24, 337

Silin, V. P., \& Tikhonchuk, V. T. 1970, J. Appl. Mech. Tech. Phys., 11, 922

Spitkovsky, A. 2004, Young Neutron Stars and Their Environments, held as part of the IAU General Assembly, 14-17 July, 2003 in Sydney, Australia, ed. F. Camilo, \& B. M. Gaensler (San Francisco, CA: ASP), IAU Symp., 218, 357 Spitkovsky, A., \& Arons, J. 2002, ASP Conf. Ser., 271, 81 